\title{
Design and Optimization of an Energy Manager for an Office Building
}

\author{
Kristin Majetta ${ }^{1 *}$, Christoph Clauß ${ }^{1}$, Jürgen Haufe ${ }^{1}$,Stephan Seidel ${ }^{1}$,Torsten Blochwitz ${ }^{2}$, \\ Edgar Liebold ${ }^{3}$, Ullrich Hintzen ${ }^{4}$, Volker Klostermann ${ }^{5}$ \\ ${ }^{1}$ Fraunhofer IIS EAS, Zeunerstraße 38, D-01069 Dresden, Germany; *kristin.majetta@eas.iis.fraunhofer.de \\ ${ }^{2}$ ITI GmbH, Schweriner Straße 1, D-01067 Dresden, Germany \\ ${ }^{3} \mathrm{NSC}$ GmbH, Äußere Zwickauer Straße 8, D-08064 Zwickau, Germany \\ ${ }^{4}$ FASA AG, Marianne-Brandt-Straße 4, D-09112 Chemnitz, Germany \\ ${ }^{5}$ Provedo GmbH, Mottelerstraße 8, D-04155 Leipzig, Germany
}

Simulation Notes Europe SNE 25(2), 2015, 99 - 108

DOI: $10.11128 /$ sne.25.tn.10297

Received: August 10, 2015 (Selected ASIM STS 2015

Postconf. Publ.); Accepted: August 15, 2015;

Abstract. Nowadays saving energy in the building sector is more important than ever. To achieve a reduction of energy consumption and also $\mathrm{CO} 2$ emissions of buildings, energy efficient Building Energy Management Systems (BEMS), respectively called energy managers, are developed in the research project enerMAT. Therefore, enerMAT creates a novel approach for simulation, optimization, and verification that will aim to design a new generation of energy-aware optimized BEMS which will allow an overall cross-trade automatic control of energy flows to maintain user comfort whilst minimizing energy consumption and $\mathrm{CO} 2$ emission.

The optimization referenced to energy uses a modelbased approach with an overall building system model enabling the assessment of the energy performance for different design and operation alternatives of the energy manager in interaction with the building. This system model allows a simulation-based, energy-aware, global, dynamic, multi-criterial optimization of the energy manager. In this paper, the idea, the modeling of an office building and its energy manager and different optimization studies and their results are presented.

Keywords: Building, Energy Management, Green Building Library, FMI, PSO Optimization

\section{Introduction}

To save energy with the aim to not exhaust the not renewable sources (oil, gas, coal) is uncontradictedly one of the most essential tasks. One of the important energy "consumers" are buildings.

For heating, cooling, air conditioning, lightening and personal electronic devices, buildings consume more than one third of primary energy at all [1]. To decrease that part a lot of ideas were created. Besides passive measures (insulation) a promising way is consuming energy in buildings more ingenious than in the past. Energy shall only be consumed if it is absolutely nesessary. One way to achieve this is the development as well as the introduction of Building Energy Management Systems (BEMS), shortly spoken, of energy managers. Operating on a runtime system energy managers take information from the building (via sensors) with the goal to calculate the energetically aware actions by controlling e.g. heating, cooling, air conditioning (HVAC).

At the ASIM workshop 2014 in ReutlingenRommelsbach we presented an approach of the development of energy managers which is investigated in the research project enerMAT [2]. Starting with an overall model of building, HVAC, ambience and user behavior an energy manager is designed (e.g. using state machines) which possesses some parameters. These parameters are used to adapt the energy manager in such a way that it is optimal regarding a cost function which bases on the energy consumption. The parameter adaption employs optimization techniques. Once the energy manager is developed it can be transformed to a runtime system without any manual interaction. 
In the enerMAT research project there are three demonstrator examples: a conference room, an office building, and a residential building. The above mentioned paper covers the conference room, whereasthis paper is focused to the office building demonstrator.

The office building (Figure 1) is the headquarters of the enerMAT project partner FASA AG in Chemnitz. By applying the enerMAT approach the development of energy managers is shown.

\section{Modeling}

The research project enerMAT investigates a modelbased approach which means that the development of energy management systems is done under utilization of a building model. Therefore, the modeling of the office building of the project partner FASA AG will be presented in this section.

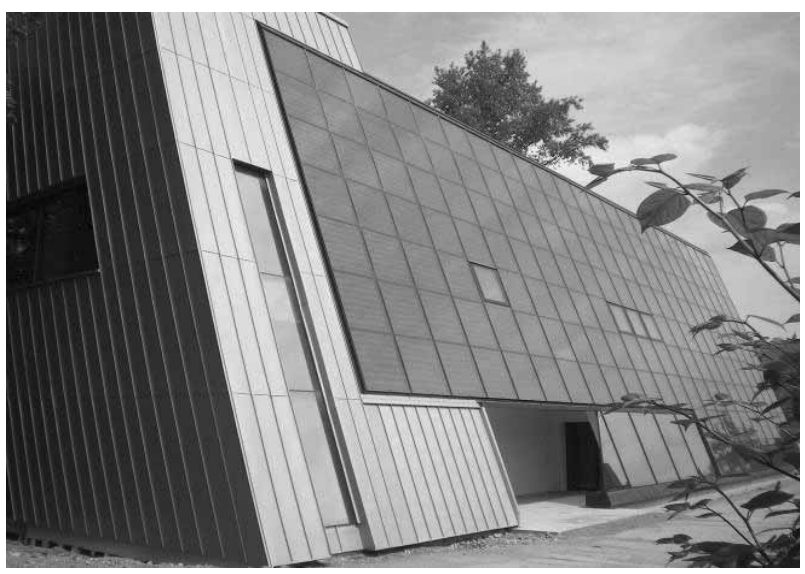

Figure 1: FASA office building in Chemnitz.

The complex model of the FASA office building demonstrator comprises the building "itself", underfloor heating, heat 'generation' (solar panel, boiler, heat pump, stove), control devices (UVR[3]), ambience (weather), user behavior, and the energy manager to be developed.

Modeling bases on the GreenBuilding library [4] which is developed by EA Systems Dresden. The library contains a large set of models for the development of energy systems with energy supply and storage systems, e.g. building component models, heating system models, heat pump models and ambience models. Since the GreenBuilding library is based on the free, object oriented, equation based Modelica language [5] it is possible to develop additional models that are required in the project but are not part of the GreenBuilding library. The models are simulated using SimulationX that is developed by the ITI GmbH.

From energetic point of view the office building consists of two parts, the energy producing and the energy consuming part. The energy producing side (shown in Figure 2) consists of a solar heating system, a stove and a heat pump. All those components are heating up the water inside a $110 \mathrm{~m}^{3}$ buffer. The water of the buffer is temperature depending layered.

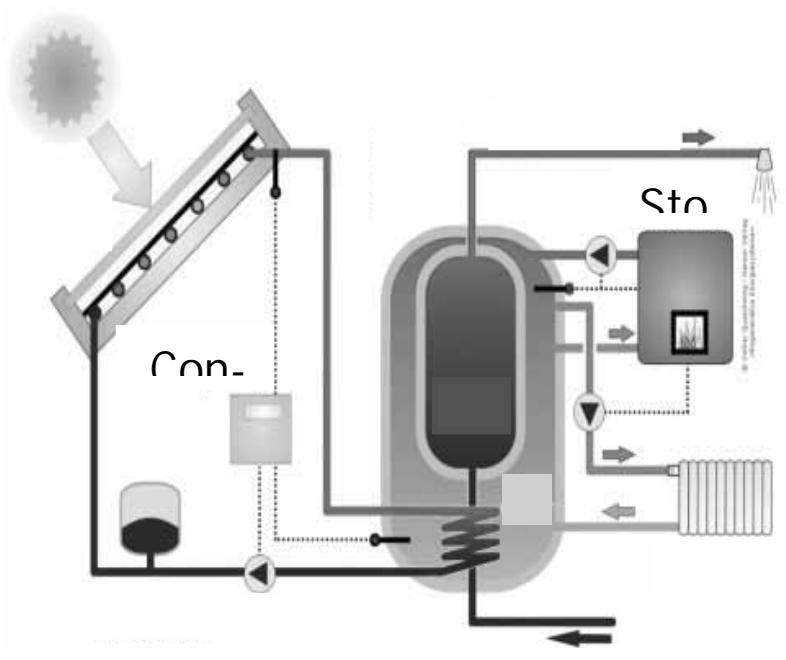

Figure 2: Energy producing part of the office building.

The energy generating system is controlled via the UVR1611 (Universalregelung 1611) which is a controller developed by the Austrian company Technische Alternative.To use the functionality of the UVR in the energy supply model, the UVR function blocks were modeled in Modelica and stored in a Modelica library.

On the energy consumption side the warm water of the buffer is used to heat the offices in the building via a floor heating system. The offices are modeled with the BuildingZone model of the GreenBuilding Library. The BuildingZone is a prepared, complex one-point model of a room, which calculates the heat losses taking ambience conditions, heat sources and losses into account. The modeler has to parametrize the zone with e.g. wall properties, room size or geographical direction. Similar office rooms were combined to a bigger building zone. The so developed model of the consumption side contains 25 building zones and needs about 5 hours for a one-year-simulation. Since this is far too long for later optimization, the 25 building zones were combined to two building zones which are representing the ground floor and the first floor of the office building. 
The models for the energy generating and the energy consuming part are connected to the complete building model which helps developing the energy manager for the office building.

To round the complete building model off, a model representing the users' behavior is added. This model gives information about the presence of the employees on working days. This knowledge is used to specify users thermal comfort ranges that's compliance is ensured by the energy manager.

\section{Energy Manager Development}

By means of the complete model of the office building two building energy management systems are developed, one for the energy producing side and one for the energy consuming side. This separation is reasonable since the aim of the energy supply side is to maximize the solar earning over the year and the aim of energy consuming side is to minimize the energy usage.

A BEMS (energy manager) prescribes set points as well as parameters of local controllers by keeping their structures. In the literature several kinds of BEMS are known e.g. rule based BEMS[6], BEMS that are based on artificial neural networks [7], fuzzy logic [8], optimization [9] or ontologies [10] and context-aware BEMS[11].

enerMAT prefers UML statecharts (Unified Modeling Language) as a basic design approach of BEMS since states are induced naturally, like, in case of a temperature, 'too cold' or 'too hot'. Since the aim of the BEMS is controlling the set points of local HVAC devices, at each state of the statechart valid set points have to be calculated.

\subsection{Energy manager for energy consumption}

Most of the energy is consumed in the office rooms. Since minimizing the whole energy consumption will be achieved by minimizing the energy consumption of a single room, the energy manager is developed for one office room. Two different approaches were investigated.

The first approach is to work with lookup tables that contain all states that the room can have under defined surrounding conditions and values of the controlling variables to achieve a certain goal.
Such a table could, for example, contain temperature ranges for the ambient temperature and for the start value of the room temperature. The aim would be to know when to turn on the heating system to achieve a certain room temperature which means, it must be known how much time the room will need to reach the target temperature.

An example is shown in Table 1. Although the values in Table 1 fictitious, they are inspired by real values which are results of estimating another one-zone-room model developed with the GreenBuilding Library.

\begin{tabular}{|c|c|c|}
\hline Ambient temp. & Start room temp. & $\begin{array}{l}\text { Heating up } \\
\text { time }\end{array}$ \\
\hline \multirow{4}{*}{$<-5^{\circ} \mathrm{C}$} & $<0^{\circ} \mathrm{C}$ & $10 \mathrm{~h}$ \\
\hline & $0^{\circ} \mathrm{C}-10^{\circ} \mathrm{C}$ & $8 \mathrm{~h}$ \\
\hline & $10^{\circ} \mathrm{C}-20^{\circ} \mathrm{C}$ & $5 \mathrm{~h}$ \\
\hline & $>20^{\circ} \mathrm{C}$ & $4 \mathrm{~h}$ \\
\hline \multirow{4}{*}{$-5^{\circ} \mathrm{C}-15^{\circ} \mathrm{C}$} & $<0^{\circ} \mathrm{C}$ & $8 \mathrm{~h}$ \\
\hline & $0^{\circ} \mathrm{C}-10^{\circ} \mathrm{C}$ & $7 \mathrm{~h}$ \\
\hline & $10^{\circ} \mathrm{C}-20^{\circ} \mathrm{C}$ & $4 \mathrm{~h}$ \\
\hline & $>20^{\circ} \mathrm{C}$ & $3 \mathrm{~h}$ \\
\hline \multirow{4}{*}{$15^{\circ} \mathrm{C}-30^{\circ} \mathrm{C}$} & $<0^{\circ} \mathrm{C}$ & $7 \mathrm{~h}$ \\
\hline & $0^{\circ} \mathrm{C}-10^{\circ} \mathrm{C}$ & $2 \mathrm{~h}$ \\
\hline & $10^{\circ} \mathrm{C}-20^{\circ} \mathrm{C}$ & $1 \mathrm{~h}$ \\
\hline & $>20^{\circ} \mathrm{C}$ & $30 \mathrm{~min}$ \\
\hline \multirow{4}{*}{$>30{ }^{\circ} \mathrm{C}$} & $<0^{\circ} \mathrm{C}$ & $3 \mathrm{~h}$ \\
\hline & $0{ }^{\circ} \mathrm{C}-10^{\circ} \mathrm{C}$ & $2 \mathrm{~h}$ \\
\hline & $10^{\circ} \mathrm{C}-20^{\circ} \mathrm{C}$ & $30 \mathrm{~min}$ \\
\hline & $>20^{\circ} \mathrm{C}$ & $10 \mathrm{~min}$ \\
\hline
\end{tabular}

Table 1: Example lookup table.

During the energy managers' runtime it permanently compares the actual room conditions with the conditions in the lookup table and decides permanently whether to switch on the heating system or not.

The obvious drawback of this method is the huge amount of data that is needed already for relatively small use cases such as the investigated single office room. Besides the ambient temperature and the start value of the room temperature, there is a number of other influence values like the temperature of the adjacent rooms and the supply temperature of the floor heating system. 


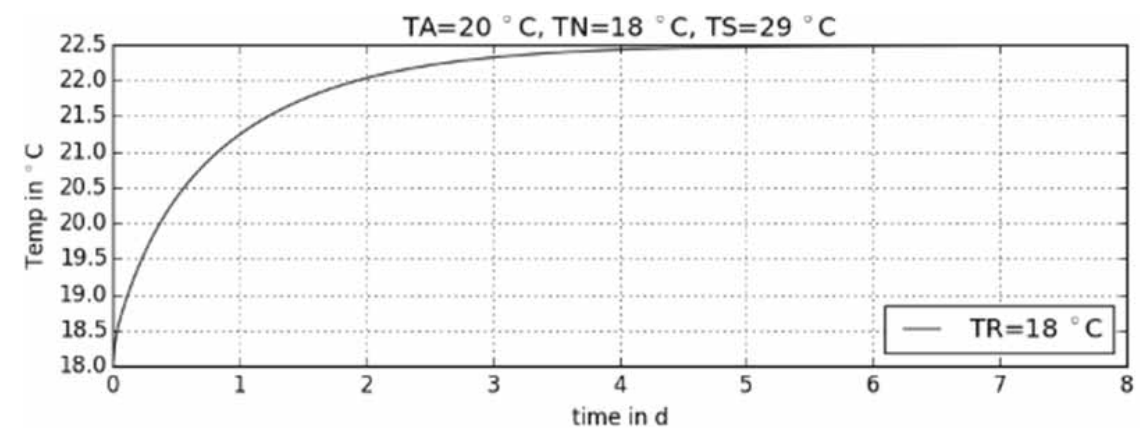

Figure 3: Heating up curve of single office room.

Since (2) has three unknowns $\left(x_{1}, x_{2}, x_{3}\right)$, three simulations under defined conditions were undertaken and the linear system $A x=b$ with

$$
\begin{aligned}
& A=\left[\begin{array}{lll}
T_{A, 1} & T_{N, 1} & T_{S, 1} \\
T_{A, 2} & T_{N, 2} & T_{S, 2} \\
T_{A, 3} & T_{N, 3} & T_{S, 3}
\end{array}\right] \\
& \text { and } b=\left[\begin{array}{l}
g_{1} \\
g_{2} \\
g_{3}
\end{array}\right]
\end{aligned}
$$

Before developing the lookup table its data items must be known. Therefore a great number of simulations had to be done to investigate the heating up time for each combination of ranges of the influence variables. Since this way of developing anenergy manager for the office room seems to be not really feasible, a second investigation was done and is shown in the following.

The simulation of one heating up process of a single office room shows, that the heating curve has the shape of an exponential function $f(t)$ (Figure 3). $f(t)$ is characterized by its steady-state value $g$, its start value $s$ and its slope $a(t=0)$ and can be described by (1):

$$
f(t)=g-(g-s) e^{\frac{-a}{g-s} t}
$$

The variables $g$ and $a$ depend on the supply temperature $T_{S}(t)$ (TS in Figure 3), the start value of the room temperature $T_{R}(t)$ (TR in Figure 3), the ambient temperature $T_{A}(t)$ (TA in Figure 3 ) and the temperature of the adjacent rooms $T_{N}(t)$ (TN in Figure 3 ).

For simplicity reasons the temperatures of the neighbor rooms are chosen to be identical.

The variables $g$ and $a$ are identified using results from particular simulations with defined values of $T_{S}(t)$, $T_{R}(t), T_{A}(t)$, and $T_{N}(t)$.

Using as an example $g$, the identification process is shown exemplarily. To identify the dependency of the steady-state value $g$ from $T_{A}(t), T_{N}(t)$ and $T_{S}(t)$, the following linear approach was chosen:

$$
g=T_{A} \cdot x_{1}+T_{N} \cdot x_{2}+T_{S} \cdot x_{3}
$$

was solved. Using (2) for each value of $T_{A}, T_{N}$, and $T_{S}$, $g$ can be calculated. A similar approach is applied for identifying the parameter $a$.

The parameter $s$ does not have to be identified since it is the start value of the room temperature which simply can be taken from the simulation. Knowing $g, s$ and $a(t=0),(1)$ is parameterized during the whole simulation of the room model and calculates the points in time for turning on or off the heating system online by transforming (1) to

$$
t_{o n, o f f}(t)=-\frac{g-s}{a} \ln \left(-\frac{w-g}{g-s}\right)
$$

where $w$ is the desired target temperature in the room.

The cooling down process of the room after turning off the heating system is also described as an exponential function in the same way as the heating process is described. For technical reasons, the number of turning on and off the heating system is resticted to one per day.

Figure 4 shows the heating and cooling process of the room for a five day periode. In the first two and a half days the heating system is always turned on since the target temperatur $w$ (blue dashed curve) is not reached.

In the second two and a half days the heating system is turned on and off in a way that the room temperature (red, continous curve) reaches the target temperature exactly at the beginning of occupancy and falls below the target temperature at the end of occupancy. 


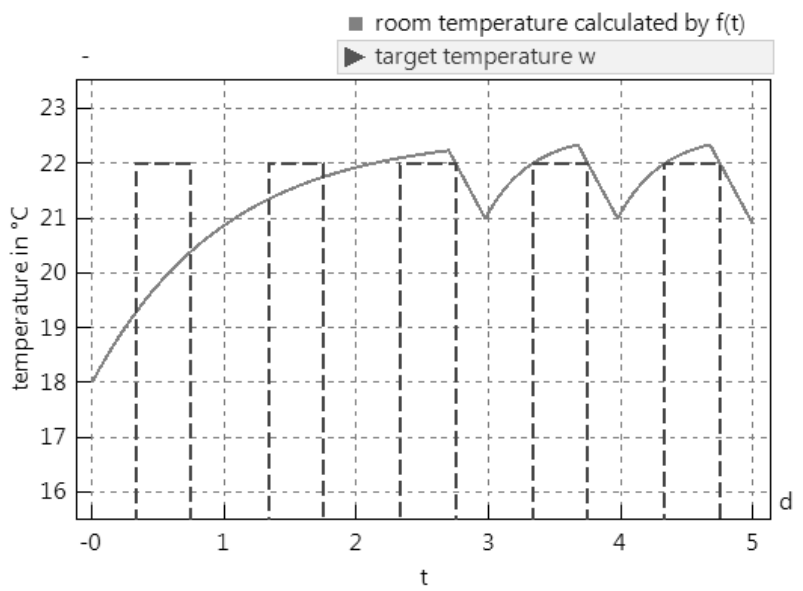

Figure 4: Heating and cooling of the office room (room temperature calculated by $f(t)$ ).

The switching points for the heating system belong to the room temperature that is calculated by the exponential function (1). Since the aim is to develop an energy manager for a 'real' office room of the office building, in the following the calculated switching points are used to control the heating system of the GreenBuilding room model.

The results in Figure 6 show, that the room temperature of the GreenBuilding model (black dashed dotted curve) shows the expected behavior by trend. This means, that the heating period in the first two and a half days is nearly the same as for the room temperature calculated by $f(t)$ (continuous red curve) and that also the switching of the heating system can be seen in the second two and a half days.

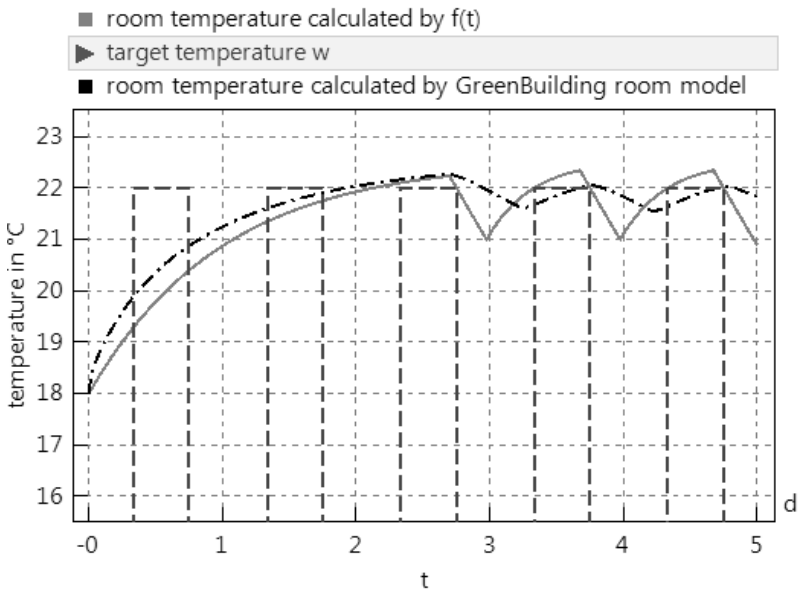

Figure 6: Comparison of room temperatures calculated by $f(t)$ and by the GreenBuilding room model.

Anyway the room temperatures are not exactly the same. A reason for that is that the exponential function (1) does not exactly represent the heating and cooling process of the room. That is because the slope of the 'real' process cannot be represented by simply parametrize the exponential function (1) with a constant slope $a(t=0)$. In fact a slope that varies in time needs to be used.

Figure 5 shows the process of the energy manager development in a schematic way.

To achieve further energy savings the lowering of the target temperature in the office room on weekends was investigated. Therefore the target temperature on weekends was set to $18^{\circ} \mathrm{C}$ (see Figure 7).

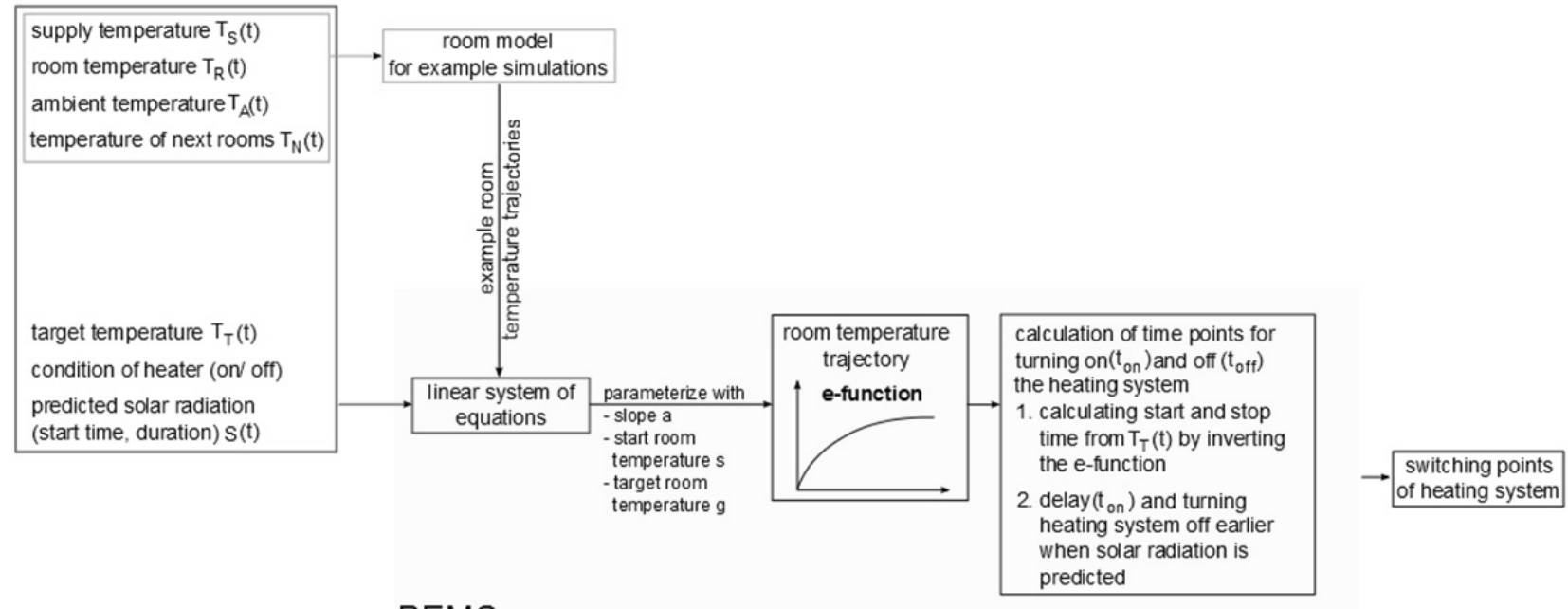

BEMS

Figure 5: Schematic description of energy manager development. 


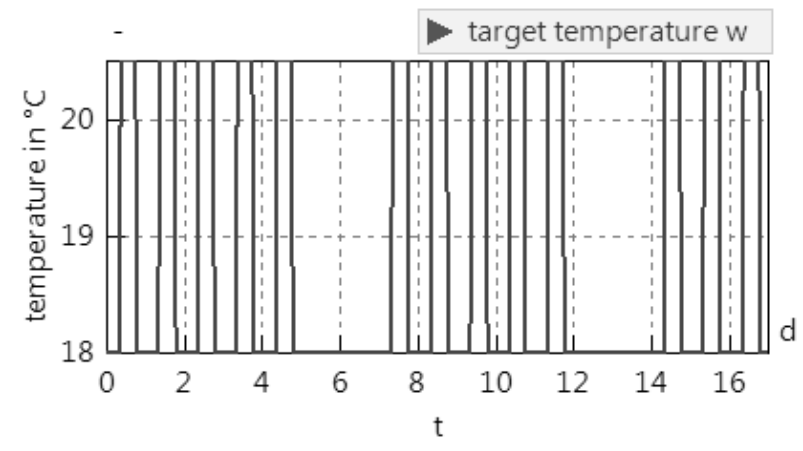

Figure 7: Target temperature $w$ with lowering at weekends.

The switching points of the floor heating system calculated by $f(t)$ lead to the temperature trajectory shown in Figure 9. It can be seen that the room temperature has a very good gradient during the week but after a weekend the heating starts too early and therefore it get too warm during the second half of the weekend. For that reason an optimization of the point in time for turning on the floor heating is described in Section 3.1.

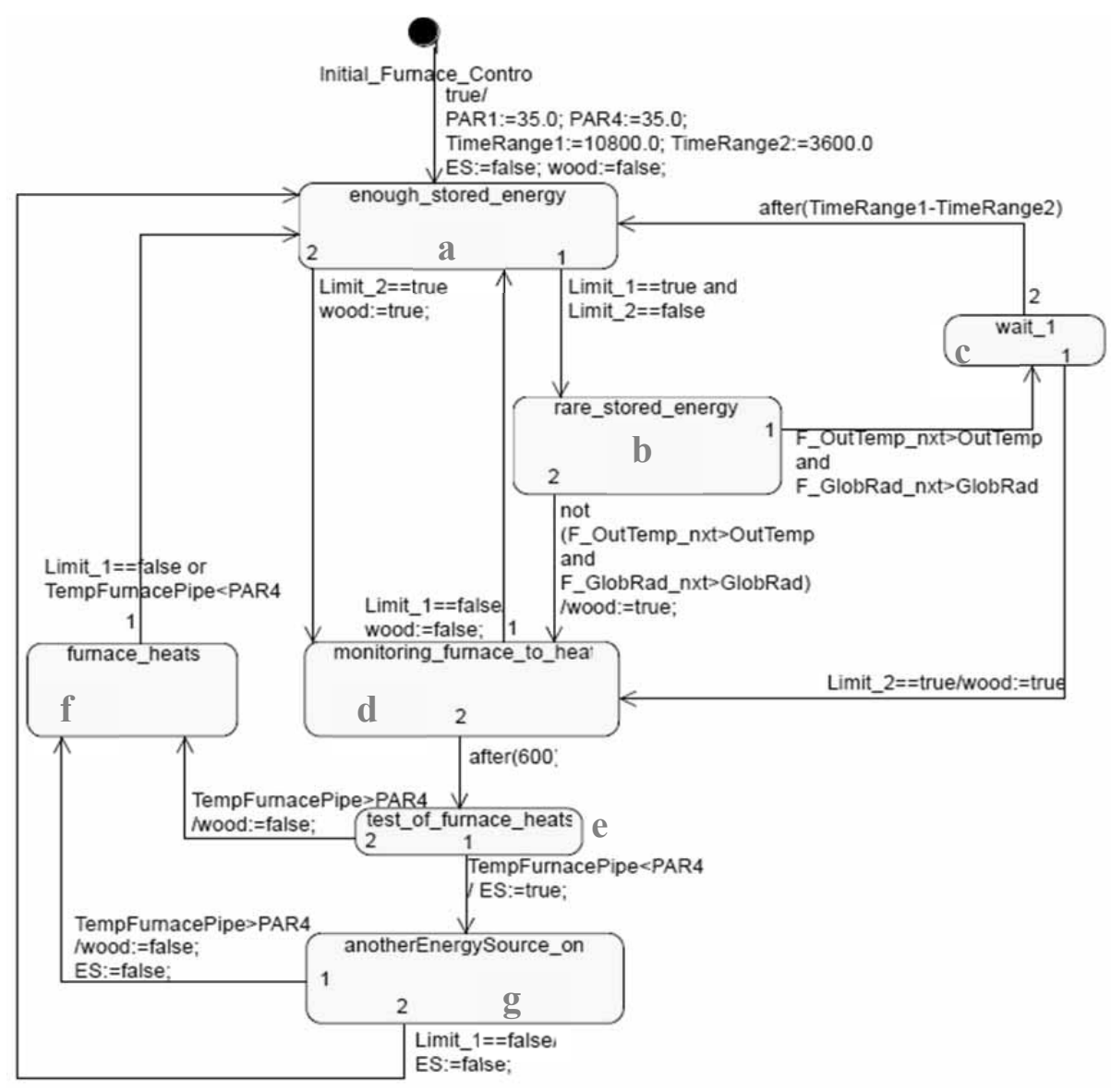

Figure 8: Statechart for controlling the stove.

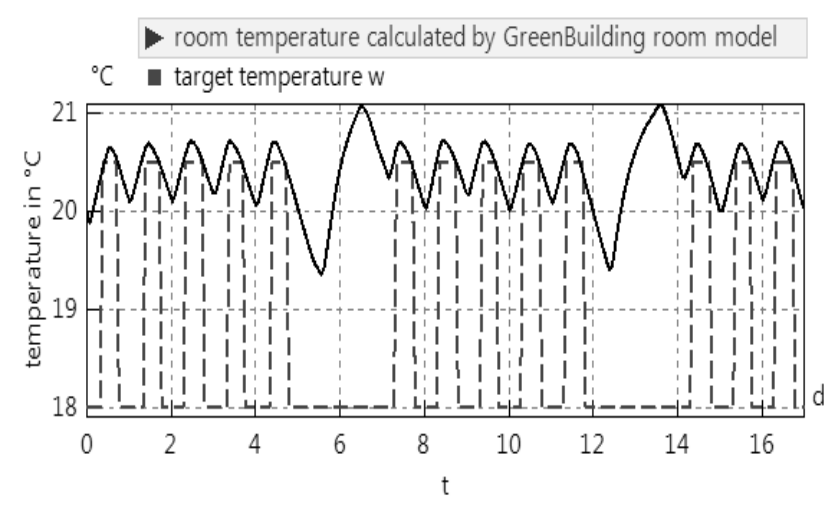

Figure 9: Temperature trajectory with setpoint lowering on weekends.

The investigations on the energy manager were all done without taking solar heating into account. The next steps must be to analyze the time delay that the heating system can be switched on later and/ or off earlier when the sun is shining into the office.

Also the prediction of the ambience conditions such as temperature and solar radiation need to be taken into account in the future. Depending on the weather conditions, the actual and predicted occupancy and the actual room temperature, the points in time for turning on and off the heating system will be optimized.

\subsection{Energy manager for energy generating}

The solar heating is already controlled by the UVR1611 controller and does not need an energy manager. Missing is the information about starting the wood-burning stove when the water in the buffer is getting too cold.

The energy manager covering that task consists of a statechart named furnace_Ctrl (Figure 9). 
The decision whether the stove is turned on depends on the actual and predicted ambience conditions like temperature and global radiation andthe needed supply temperature of the floor heating system.

If enough energy in the buffer (state a) is available, limits are checked to find out if the available energy becomes low or if heating the stove is necessary immediately. If energy is low (state b) and solar radiation is expected, it is sensible to wait (state c). If otherwise heating is needed (value wood is true, state d) it is checked whether heating has actually started (state e). If so (state f) it again is checked if enough heat is in the buffer (state a). If the stove has not started heating, it is checked if there is another energy source (state g) that could be uses to heat the water in the buffer.

To calculate the Boolean limit values (limit 1, limit 2) which indicate if there is enough heat in the buffer, a reference temperature Tref is computed (4) with the help of the adjusting parameters $c_{0}, c_{1}$ (that will be optimized later) and the outdoor temperature Tout.

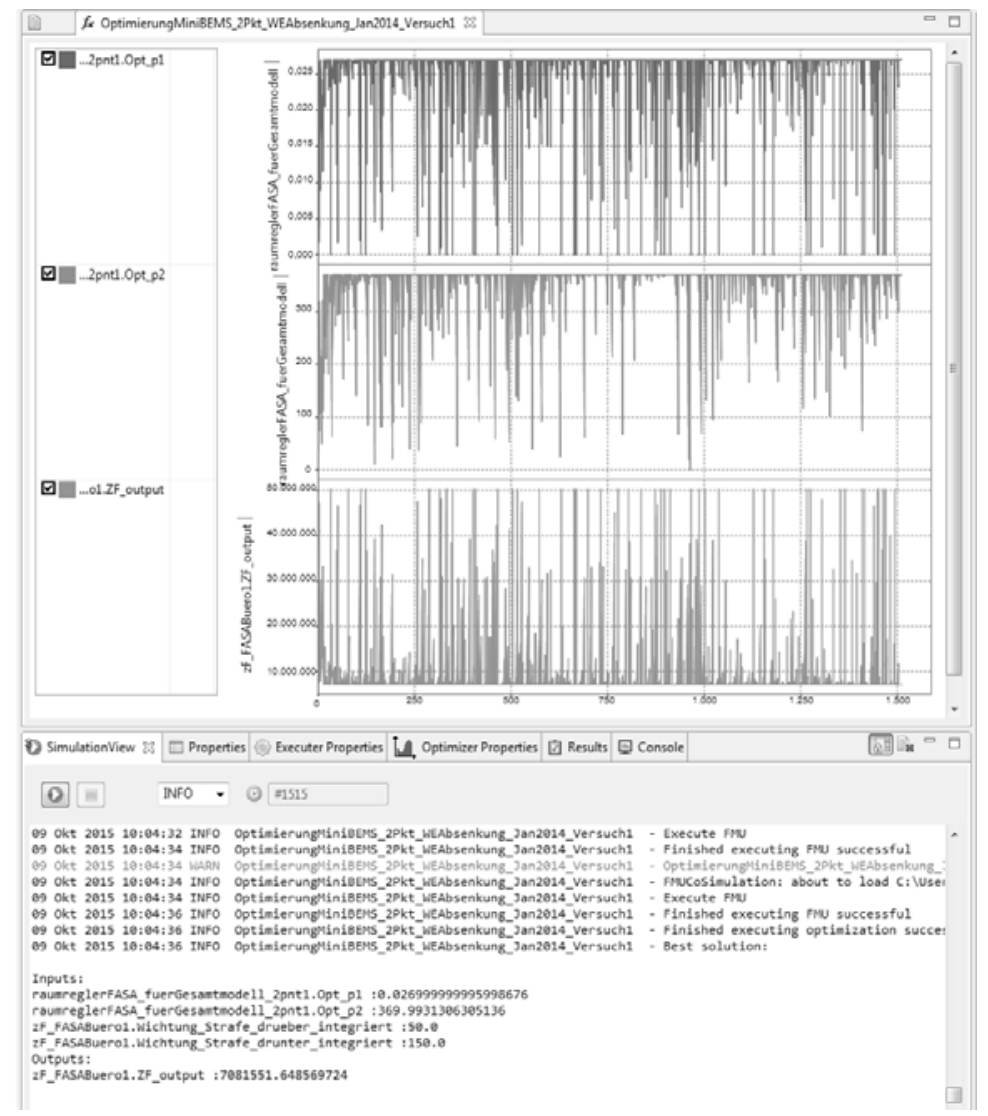

Figure 10: Optimization tool from EAS.

$$
\text { Tref }=\min \left(80, c_{0}+c_{1}(25-\text { Tout })\right)
$$

The Boolean limit values are calculated by ingTre $f$ with several underfloor heating supply temperatures $T s_{1}, T s_{2}, T s_{3}$ and $T s_{4}$ measured at the tank.

$$
\begin{aligned}
\text { limit1 }= & \left(T s_{1}<\text { Tref }+5 \text { and } T s_{2}<\text { Tref }\right) \\
\text { or } & \left(T s_{3}<\text { Tref }+5 \text { and } T s_{4}<\text { Tref }\right)
\end{aligned}
$$

Further adjusting parameters $c_{2}, c_{3}$ (that also will be optimized later) are used to calculate the burn time $B t$, which is proportional to the mass of wood to be burned:

$$
\left.B t=c_{2}+c_{3}(25-T o u t)\right)
$$

This developed energy manager for the heat generating side of the office building is simulated and optimized together with the developed building models as shown in section 3

\section{Optimization Studies}

In this section different optimization studies and their results are shown. For the optimization the energy managers for generation and consumption are combined with a building model to complete simulation and optimization models.

These models are exported as functional mockup units (FMUs) and connected to a particle swarm optimizer via the functional mockup interface (FMI). Different optimization studies and their results are shown in this section.

\subsection{Energy manager for energy consumption}

As mentioned above the exponential function (1) does not exactly represent the heating and cooling process of the model room. This is why the points in time for turning on and off the floor heating system should be improves by optimizing.

Therefore two optimization parameters $o p t \_p 1$ and $o p t \_p 2$ were introduced that in- or decrease the time between turning the heating system on or off and reaching the target temperature $\mathrm{w}$. 
To minimize the energy consumption as well as assure comfort conditions the target function (7) was applied.

$$
T F=E+p \_b e l o w+p \_a b o v e
$$

In (7) $E$ denotes the energy consumption of the floor heating system, $p \_b e l o w$ and $p \_a b o v e$ are penalty terms that penalize the target function $T F$ when it is too cold or too warm in the room.

As mentioned the particle swarm algorithm was used for the optimization. Figure 10 shows a screenshot of the GUI of the optimization tool developed by Fraunhofer IIS EAS.

For the optimization the energy manager was connected to a room model to a complete optimization model which was exported as an FMU and connected to the optimizer via the FMI interface.

The optimized parameters are:

$$
\begin{aligned}
& \text { opt_pl }=0.027 \\
& \text { opt_p2 }=370
\end{aligned}
$$

Using these optimization parameters in the simulation of the complete model (energy manager connected to the room model) the energy consumption decreases from $120,7 \mathrm{kWh}$ to $108,2 \mathrm{kWh}$ while the comfort conditions are kept.

Figure 11 compares the simulated room temperature before and after the optimization.

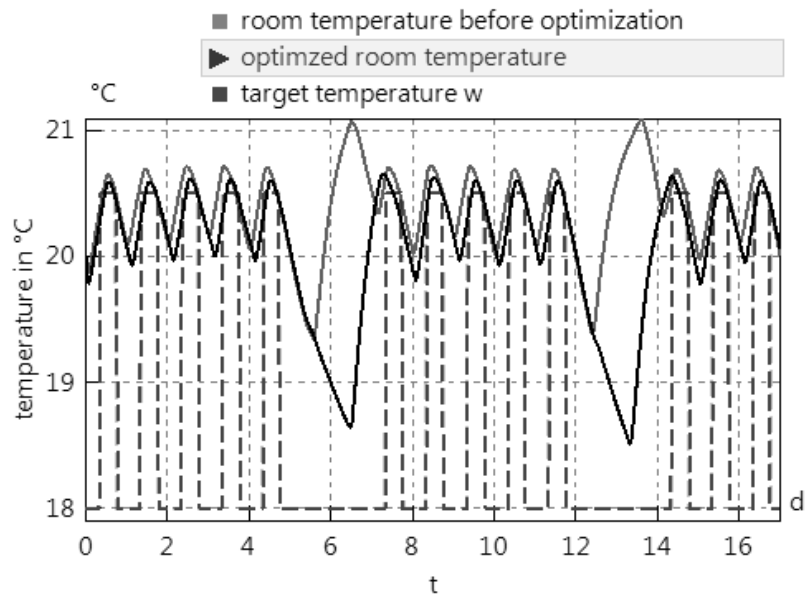

Figure 11: Room temperatures before and after optimization.

\subsection{Energy manager for energy generating}

As described in section 1, two differently detailed buildings models are available. Those models include, besides the stove, a heat pump which is not controlled by the energy manager.

When simulating the more detailed model including the developed energy manager for the first six months of the year using an arbitrarily chosen set of adjusting parameters, the energy supplied by stove and heat pump is calculated (second line in Table 2).

Unfortunately, this simulations takes around 5 hours (used hardware: Windows 7 Enterprise 64bit, Intel® Core $^{\mathrm{TM}}$ i7-4600U CPU @ 2.7 GHz, 8 GB RAM). Since this is far too much for the application of optimization methods, the less detailed model is used since its simulation time is around 5 to 10 minutes.

The results of this simulation (third line of Table 2) show that the energy consumption of the stove and the heat pump is higher compared to the simulation of the more detailed model. Nevertheless, the less detailed building model is an approximation of the detailed model which describes the building behavior in principle correctly. Since its simulation time is much shorter, it is used for the optimization of the energy manager.

A further acceleration was reached by defining the adjusting parameters $c_{0}$ and $c_{2}$ to be of the type integer during optimization instead of real. The following intervals for the adjusting parameters were defined heuristically:

$$
\begin{array}{ll}
0 \leq c_{0} \leq 30, & 0 \leq c_{1} \leq 2 \\
0 \leq c_{2} \leq 20 & 0 \leq c_{3} \leq 1
\end{array}
$$

The objective function is the sum of energy supplied by the heat pump as well as by the stove. Furthermore, penalty terms are added to ensure some temperature restrictions at the buffer.

$$
C=E_{\text {stove }}+E_{\text {heat pump }}+\text { penalty }
$$

Most important is a low energy consumption of the heat pump since it uses electric power that is expensive compared to the wood for the stove. The optimization results for the parameters and energy consumptions are shown in line 4 in Table 2 . It can be seen, that the optimized adjusting parameters lead to a lower energy consumption compared to the simulation of the less detailed model with arbitrary parameters (line 3 of Table 2). 
The optimized adjusting parameters are now used to simulate the original, more detailed model (line 5 in Table 2) and the results show that the optimized parameters of the less detailed model also lead to better results in the more detailed model (second line in Table 2). This gives the conclusion, that the model simplifications that led from the more detailed model to the less detailed one were allowable.

\begin{tabular}{|l|l|l|l|l|l|l|}
\hline & $c_{0}$ & $c_{1}$ & $c_{2}$ & $c_{3}$ & $\begin{array}{l}\text { Ener- } \\
\text { gy } \\
\text { in } \\
\text { kWh }\end{array}$ & $\begin{array}{l}\text { Ener- } \\
\text { gy } \\
\text { heat } \\
\text { pump } \\
\text { in } \\
\text { kWh }\end{array}$ \\
\hline $\begin{array}{l}\text { detailed } \\
\text { model }\end{array}$ & 30 & 1 & 18 & 0.5 & 115 & 0 \\
\hline $\begin{array}{l}\text { less de- } \\
\text { tailed } \\
\text { model }\end{array}$ & 30 & 1 & 18 & 0.5 & 425 & 1421 \\
\hline $\begin{array}{l}\text { less de- } \\
\text { tailed } \\
\text { model } \\
\text { optimized }\end{array}$ & 30 & 1.97 & 20 & 1 & 310 & 626 \\
\hline $\begin{array}{l}\text { more } \\
\text { detailed } \\
\text { models } \\
\text { with op- } \\
\text { timized } \\
\text { parame- } \\
\text { ters }\end{array}$ & 30 & 1.97 & 20 & 1 & 94 & 0 \\
\hline
\end{tabular}

Table 2: Optimization studies.

As could be seen during the optimization work, the performance of one simulation run can be quite bad since building models naturally are relatively extensive. Therefore, it is often too time consuming to finish optimization runs within reasonable time.

To overcome these difficulties, several strategies can be applied:

\section{- Take simplified models instead of accurate ones.}

It is possible to over-simplify models. Therefore, it needs to be investigated, how simple a model can be to generate still reasonable optimization results. At least the following consideration is useful: If simplified models are used for optimization, the parameters obtained from optimization should be applied to the more accurate model. Such a verification simulation should prove that the parameters still produce good results.
- Reduce the number of parameters during optimization.

A low number of parameters is advantageous for optimization runs. Parameters which have not a great influence on the optimization result can be identified by performing a sensitivity analysis.

\section{- Chose suitable time intervals for the parameter optimization.}

Often some states become active within dedicated time intervals only. Therefore, it is sometimes possible to optimize groups of parameters separately within shorter time intervals. This improves the performance. E.g. typically parameters which influence heating devices should not be optimized within summer months.

\section{- Do not simulate unreliable parameter values.}

Sometimes, the optimization algorithm choses unreliable as well as not realistic parameter constellations, which cause bad performant simulations or simulation crashes. Such obviously bad parameter constellations should be selected before simulation. This selection can be included into the simulation model.

\section{- Use parallelization.}

Both optimization as well as simulation can be accelerated by parallelization.

- Choose good process parameters of the optimization method.

The optimization method can be adapted by suitable optimization process parameters. This influences the performance of optimization drastically.

\section{Summary}

During the enerMAT project different BEMS respectively energy managers were developed. Since enerMAT follows a model based approach, the modelling of the FASA office building (two differently detailed models) was introduced. Additionally the development of two energy managers for the FASA office building was presented in this paper.

The energy manager for the energy generating part is developed as a statechart with adjusting parameters that are optimized to reach minimal energy consumption. 
The energy manager for the heat consuming part was developed by process identification of the heating and cooling process using the example of one room. A bottleneck in this process is the often bad simulation and optimization performance. Therefore some strategies are discussed to improve the performance.

The investigation shows that a simple control algorithm can be adapted to a special room by optimization if suitable optimization parameters are introduced. The better the control algorithm is the less optimization parameters will change when other test cases (e.g. ambient conditions) are applied. This way the robustness as well as the quality of a control algorithm can be checked.

The potential of this approach is by far not exhausted yet: The optimization can comprise both adjusting parameters of the energy manager as well as constructing parameters of the building. Thus more common target functions are possible. Other investigations are necessary to support the development of the statechart.

\section{Acknowledgement}

\begin{tabular}{|l|l|}
\hline $\begin{array}{l}\text { The research is } \\
\text { funded by }\end{array}$ & Gefördert durch: \\
& $\begin{array}{l}\text { Bundesministerium } \\
\text { für Wirtschaft } \\
\text { und Technologie }\end{array}$ \\
& $\begin{array}{l}\text { aufgrund eines Beschlusses } \\
\text { des Deutschen Bundestages } \\
\text { Förderkennzeichen: 03ET1084 }\end{array}$ \\
\hline
\end{tabular}

\section{References}

[1] EU Energy and transport in figures, statistical pocket book 2007/2008

[2] Clauss C, Haufe J, Blochwitz T et al.. Model Based Optimization of Building Control Systems. Proceedings of the ASIM-Workshop STS/GMMS 2014; 2014 Feb 20-21; Reutlingen-Rommelsbach, Germany; 2014, P. 131-138.

[3] Website of the Austrian company "Technische Alternative" :

http://www.ta.co.at/de/produkte/uvr1611/freiprogrammierbare-regelung-uvr1611.html

[4] Unger R, Schwan T, Mikoleit B et. al.. „Green Building“ - Modelling reneable building energy systems and electric mobility concepts using Modelica. Proceedings of the $9^{\text {th }}$ International Modelica Conference; 2012 Sep 3-5; Munich, Germany; 2012, P. 897-906.

[5] www.Modelica.org

[6] Doukas H, Patlitzianas KD, Iatropoulos K et al.. Intelligent building energy management system using rule sets. Building and Environment; 2007; 42(10):35623569 .

[7] Kalogirou SA. Applications of artificial neural-networks for energy systems. Applied Energy; 2000; 67:17-35.

[8] Kolokotsa D, Stavrakakis GS, Kalaitzakis K et al.. Generic algorithms optimized fuzzy controller for the indoor environmental management in buildings implemented using PLC and local operating networks. Engineering Applications of Artificial Intelligence; 2002; 15(5):417-428.

[9] Castilla M, Álvarez JD, Berenguel M et.al.. A comparison of thermal comfort predictive control strategies. Energy and Buildings; 2011;43:2737-2746.

[10] Rosselló-Busquet A, Brewka LJ, Soler J et. al.. OWL Ontologies and SWRL Rules Applied to Energy Management. UKSim $13^{\text {th }}$ International Conference on Modelling and Simulation; Emmanuel College Cambridge, United Kingdom; 2011, P. 446-450.

[11] Byun J, Park S. Development of a Self-adapting Intelligent System for Building Energy Saving and Contextaware Smart Services. IEEE Transactions on Consumer Electronics; 2011; 57(1). 